\title{
Does Access to Health Care Impact Survival Time after Diagnosis of AIDS?
}

\author{
JOLYNN PRATT MONTGOMERY, Ph.D., M.P.H., ${ }^{1}$ BRENDA W. GILLESPIE, Ph.D., ${ }^{2}$ \\ ANNE C. GENTRY, M.P.H., ${ }^{1}$ EVE D. MOKOTOFF, M.P.H., ${ }^{1}$ LAWRENCE R. CRANE, M.D., ${ }^{3}$ \\ and SHERMAN A. JAMES, Ph.D. ${ }^{4}$
}

\begin{abstract}
Lack of access to health care is often blamed for poor health outcomes, but this effect is not supported by existing HIV/AIDS literature. The authors examined the association between access to care and survival time after progression to AIDS, using survival analysis methods. This study combined data from two CDC sponsored studies of HIV-infected persons, a crosssectional interview study and a longitudinal medical record review study. Study subjects included 752 persons who progressed to AIDS before December 31, 1999, and were patients at either of two major HIV care facilities in Detroit, MIchigan. Separate statistical models were used to test associations between survival time after meeting the criteria for AIDS and two indicators of access to health care: (1) perceived access to health care and (2) health care utilization patterns. Perceived access was not associated with survival time after AIDS, but patterns of health care utilization were significantly associated with survival time after AIDS $(\mathrm{HR}=2.04, p<0.001)$. Individuals who received a greater proportion of their care in the ER had a worse survival prognosis than those who received more of their health care in an outpatient clinic setting. It is crucial that we provide $\mathrm{HIV}$-infected populations with tools that enable them to access a regular source of health care.
\end{abstract}

\section{INTRODUCTION}

D IFFERENCES IN SURVIVAL TIME of HIV infected persons are often attributed to differences in access to health care. ${ }^{1,2}$ While this explanation is logical and supported for other disease processes, there is little evidence in the literature to support the conclusion that better access to health care improves survival probability in people living with AIDS. Some stud- ies have shown that perceived access to health care was associated with health-related quality of life measures. ${ }^{3,4}$ It is important to study the factors that give rise to differences in survival time in the United States so that survival can continue to improve. The recent survival gains, which are often associated with newly available antiretroviral drugs, differ significantly among blacks, whites, men and women. ${ }^{5,6}$ If the factors responsible for these

\footnotetext{
${ }^{1}$ Michigan Department of Community Health, HIV/AIDS Surveillance Section, Detroit, Michigan.

${ }^{2}$ Center for Statistical Consultation and Research, University of Michigan, Ann Arbor, Michigan.

${ }^{3}$ Wayne State University, Detroit Medical Center, Department of Internal Medicine, Division of Infectious Diseases, Detroit, Michigan.

${ }^{4}$ University of Michigan, Ann Arbor, Departments of Epidemiology and Health Behavior \& Health Education, Ann Arbor, Michigan.
} 
differences can be determined, they may then be modified.

Drug therapies from the mid-1990s changed the picture of the AIDS epidemic in the United States. The availability of new antiretroviral drugs and highly active antiretroviral therapy (HAART) in the United States since 1996 has lead to a significant reduction in HIV-related morbidity and mortality. ${ }^{7-10}$ Incidences of AIDS-defining opportunistic illnesses have significantly decreased with the introduction of these combination therapies. 5,11 Clearly, access to these medications extends survival time, but antiretrovirals are very expensive. Private insurance providers, health maintenance organizations, and public programs offer assistance with the cost of these drugs. Attempts to target under-represented populations for involvement in clinical trials for new antiretroviral drugs through the Terry Beirn Community Programs for Clinical Research on AIDS (CPCRA) have also helped to close the gap in access to HIV-related treatment. ${ }^{1}$

In this article, we examine perceived access to health care and utilization of health services and their associations with survival after AIDS, controlling for HAART status. We hypothesized that indicators of access to health care (both perceived access to care and health care utilization) were significantly associated with survival.

\section{METHODS}

This study was conducted under the auspices of the Michigan Department of Community Health's HIV / AIDS Surveillance Section, located in Detroit, Michigan. The databases from two surveillance studies were matched: the Adult/Adolescent Spectrum of HIV Disease (ASD) project, and the Supplement to HIV and AIDS Surveillance (SHAS) project. Both ASD and SHAS are ongoing multi-site projects, located throughout the United States. This study examines data from the two Detroit, Michigan sites. These facilities offer inpatient, outpatient, and emergency care for HIV-infected patients, and are the major HIV care providers in Southeastern Michigan. Candi- dates for inclusion in this project were at least 18 years of age at the time of their SHAS interview, had completed a SHAS interview, and had an ASD record.

ASD is a surveillance study, initiated in $1990 .{ }^{12}$ Data from all medical records available at the two Detroit sites were abstracted in 6month intervals from the time an HIV-infected person presented for care at one of the participating facilities until he/she died or was lost to follow-up. Medical records for any visit to these facilities were included. Information was collected on HIV-related illnesses, other medical conditions, drug therapies, laboratory tests (including CD4 counts), and health care utilization. At one site, all HIV-infected men and women who presented for care were included in ASD. At the other site, all HIV-infected women but only $40 \%$ of HIV-infected men were included in ASD, owing to a large number of male patients. Between January, 1990 and December, 1999, approximately 4,600 patients were included and followed as part of the Detroit ASD study.

Initiated in 1990, SHAS is a cross-sectional interview-based surveillance project of persons with HIV or AIDS reported to the state health department. ${ }^{13}$ The SHAS study collected information on demographics, health insurance, socio-economic status, sexual behavior, drug use, reproductive history, and adherence to drug therapies. SHAS candidates were identified as HIV positive at the care facilities and were approached for an interview by a full-time, trained SHAS interviewer. Informed consent was obtained before all interviews. The refusal rate for SHAS was approximately $2 \%$. Between June, 1990 and December, 1999, approximately 2,000 patients were interviewed as part of the Detroit SHAS study.

In Michigan, ASD and SHAS are linked to the HIV/AIDS Reporting System (HARS). HARS is the surveillance registry that contains all reported cases of HIV and AIDS. The ASD/SHAS matched database was created by merging ASD and SHAS based on a numerical identifier that links both studies to HARS. The ASD and SHAS subjects were demographically similar to HIV and AIDS cases in the Detroit metropolitan area (ASD was 72\% male, 70\% 
black, and $65 \%$ age $20-40$; SHAS was $77 \%$ male, $74 \%$ black, and $64 \%$ age $20-40$; HARS in the Detroit metropolitan area was $78 \%$ male, $69 \%$ black, and $62 \%$ age $20-40$ ). Institutional Review Board approval or exemption from review was granted for ASD, SHAS, and the matched database study. The ASD/SHAS matched database included records for 1550 people.

Combination therapy (HAART), which includes three classes of antiretrovirals [nucleoside reverse transcriptase inhibitors (NRTI), protease inhibitors (PI), and non-nucleoside reverse transcriptase inhibitors (NNRTI)], has had a significant impact on survival time in people living with AIDS. In 1996, the FDA approved the first protease inhibitor through its fast track approval process. While protease inhibitors became available in 1996, the United States Public Health Service did not establish treatment guidelines, which recommended triple drug therapy, until $1998 .{ }^{14}$ Since specific regimens and dates of antiretroviral usage were not collected in ASD until 1998, we were limited in our ability to categorize HAART. We defined HAART as prescription of at least three antiretroviral drugs in a specified 6-month interval, whether or not these were prescribed simultaneously. For this study, subjects were divided into the following two categories: (1) those who were not prescribed HAART, as defined for this study (the "no HAART" group) and (2) those who have ever been prescribed HAART, as defined for this study (the "HAART" group). Every person in this study fits into one of these two mutually exclusive categories.

The health outcome variable examined was survival time. For the purpose of obtaining a relatively consistent beginning point for each person, survival was measured from the date a person first met the criteria for AIDS to time of death. A consistent AIDS definition was used for all persons to avoid the bias that would result from a sudden increase in the number of AIDS cases in 1993 when the AIDS definition was expanded. For all persons, the 1993 AIDS case surveillance definition ${ }^{15}$ was used, which includes laboratory-based criteria (CD4 count $<200$ or $<14 \% / \mu \mathrm{L}$ of blood) and clinical criteria (AIDS-defining opportunistic illnesses). For persons in ASD before 1993, the 1993 case definition could still be applied because all necessary data (CD4 count, pulmonary $\mathrm{TB}$, invasive cervical cancer, and recurrent pneumonia) were available since the ASD study began in 1990.

Vital status was collected for the ASD database from the following sources: (1) medical record documentation of death, (2) information from Michigan and Detroit death registries, and (3) report of AIDS-related deaths to the Michigan HARS from other hospitals (those not participating in ASD). Because we made an extensive search of death records, we assumed that any patient not known to have died, or to be lost to follow-up, was alive as of December 31,1999 . Those who were lost to follow-up before December 31, 1999 were censored as of the date of their last visit.

Two separate survival analysis models were used, one that included indicators for perceived access to health care and one that included an indicator for utilization of health care services. The health outcome variable in both models was survival time after AIDS, and race, sex, and mode of transmission were included as predictor variables. Greater than $97 \%$ of the subjects reported belonging to either black or white racial groups, therefore other racial and ethnic categories were excluded from these analyses. Age at time of AIDS was included as a continuous variable. The first survival analysis model examined perceived access to health care based on three questions: (1) In the past 12 months, have you ever been denied medical care for HIV infection or AIDS because you could not pay for treatment (yes/no)? (2) In the past 12 months, have you not sought medical care for HIV infection or AIDS because you couldn't pay for it (yes/no)? (3) Do you currently have health care insurance, including government-sponsored insurance such as Medicaid (yes/no)? Responses to these questions from the SHAS interview were used as dichotomous variables for the survival analysis models.

The second survival analysis model focused on health care utilization as measured by the total number of visits to the emergency room (ER) divided by the total number of visits to 
both the ER and outpatient clinics. There were no patients in this study who sought care exclusively through the emergency department. This ratio demonstrates the care-seeking behavior of each individual. A person who used the ER as a primary source of health care had a very different care-seeking profile than a person who utilized a regular health care provider and who sought regular outpatient appointments.

We used the Centers for Disease Control and Prevention (CDC) defined mode of transmission categories ${ }^{16}$ : (1) Men who have sex with men who are also injection drug users (MSM/IDU), (2) men who have sex with men (MSM), (3) injection drug users (IDU), (4) recipients of blood products (transfusion, organ transplant, hemophilia related blood products, etc.), (5) individuals who engage in heterosexual sex, and (6) those with no identified risk (NIR). These categories of risk were applied hierarchically, resulting in mutually exclusive categories. ${ }^{16}$ For the purpose of these analyses, MSM/IDU, blood products, and heterosexual sex were combined into one category (referred to as "other"). Preliminary analyses (not shown) demonstrated that these modes of transmission had relatively small sample sizes and were not significantly associated with survival. Dummy variables for IDU, NIR and "other" (compared to MSM) were used for the survival models. MSM was generally used as the referent category for mode of transmission in the literature. ${ }^{17-21}$

Cause of death was not examined, because it was not available for all cases known to have died. However, cause of death was available for $58 \%$ of deaths in the ASD database (of which this study was a subset). Of those with a known cause of death, approximately $87 \%$ were HIV-related. The number of deaths not due to HIV-related causes were evenly distributed between the treatment groups, suggesting that the probability of death being HIV-related did not change significantly with HAART.

Cox proportional hazard models were used to examine the association between predictor variables and survival time, with ties handled by Efron's method. Only post-SHAS questionnaire survival time was considered, and survival analysis methods for delayed entry (i.e., left truncation) were employed. ${ }^{22}$ Log minus log survival curves were plotted against time for each covariate to determine the appropriateness of the proportional hazards assumption. ${ }^{22}$ Where the proportional hazards assumption was not appropriate, several time-dependent functions were tested to find the model that best fit the data. ${ }^{23}$ Interactions between variables in the models were tested and $t$-tests were used to check for colinearity. Data analyses were performed using SAS Software and S-Plus. ${ }^{24,25}$

\section{RESULTS}

Of the 1,550 subjects included in the ASD/SHAS matched database between January, 1990 and December 1999, this study included the 752 people who met the definition for AIDS, and for whom complete data were available for the variables examined. Approximately $35 \%$ of subjects had received HAART, $72 \%$ were black, $78 \%$ were male, and $54 \%$ were MSM. Responses to the access to health care questions were distributed as follows: $4.0 \%$ were denied medical care because they could not pay, $7.5 \%$ did not seek medical care because they could not pay, and $7.5 \%$ did not have current health insurance at the time of their SHAS interview. While there was some overlap in responses to these questions $(34 \%$ were denied care and did not seek care because they could not pay, $16 \%$ were denied care because they could not pay and had no health insurance, and $20 \%$ had no health insurance and did not seek care because they could not pay), those who were denied medical care were not necessarily the same subjects who did not seek care or did not have health insurance.

For each covariate, log minus log survival plots were examined and confirmed that hazards were proportional for race, sex, mode of transmission, the three indicators of access to care, and the health care utilization variable. However, hazards did not appear to be proportional for HAART. Multiple time-dependent models were tested to determine the best representation of the functional form of 
HAART. The time-dependent covariate used was HAART $\times$ survival time.

The methodology for defining HAART was crude, thus we tested the validity of this HAART definition using just the ASD database $(n=1,709)$. We found that HAART was associated with a threefold increase in survival time, which is similar to that described in the literature. ${ }^{7-10}$

Indicators of perceived access to health care and utilization of health care were explored in separate Cox proportional hazards models. The perceived access-based Cox model included race, sex, mode of HIV transmission, HAART, time-dependent HAART, denial of care due to an inability to pay, not seeking care because of an inability to pay, and health insurance status. The utilization-based Cox model included race, sex, mode of HIV transmission, ER visit ratio, HAART, and its corresponding time dependent covariate. For each variable, the estimate and significance were robust to changes in other covariates in the model. Table 1 shows the results from the perceived access model, and Table 2 shows the results of the utilization model.
HAART was a significant predictor of survival in both the access (Table 1) and utilization (Table 2) models, $p<0.001$. The association between HAART and survival changed over time as illustrated by a significant timedependent covariate in each model. [Hazard ratios (HR) in the access-based model: HR at 1 year $=0.12,2$ years $=0.14,5$ years $=0.23$, 8 years $=0.37$. $\mathrm{HR}$ in the utilization-based model: $\mathrm{HR}$ at 1 year $=0.12,2$ years $=0.14,5$ years $=0.22,8$ years $=0.35$.] HAART was consistently associated with improved probability of survival, although this association tended toward the null as survival time increased.

In the first survival analysis model, the perceived access to health care model (Table 1), none of the indicators of access to health care were significantly associated with survival time after AIDS. Additionally, we tested a model in which any one response indicating poor perceived access to care constituted the access variable. This model was also not significant, indicating that the lack of association was not due to inadequate power.

In the second survival analysis model, the

Table 1. Perceived Access to Health Care ${ }^{a}$

\begin{tabular}{|c|c|c|}
\hline Predictor variable & $\begin{array}{l}\text { Hazard ratio }(\mathrm{HR}) \\
(\mathrm{n}=752 ; 552 \text { deaths })\end{array}$ & P-value \\
\hline $\begin{array}{l}\text { Denied care because unable to pay } \\
\text { (denied care vs. not denied care) }\end{array}$ & 0.69 & 0.119 \\
\hline $\begin{array}{l}\text { Not sought care because unable to pay } \\
\text { (not sought care vs. sought care) }\end{array}$ & 1.10 & 0.588 \\
\hline $\begin{array}{l}\text { Health Insurance } \\
\quad \text { (insurance vs. no insurance) }\end{array}$ & 1.01 & 0.961 \\
\hline HAART $($ referent $=$ HAART) & & $<0.001^{\mathrm{b}}$ \\
\hline Time dependent (HAART $\times$ time) & & $0.020^{c}$ \\
\hline HR at 1 year & 0.12 & \\
\hline HR at 2 years & 0.14 & \\
\hline HR at 5 years & 0.23 & \\
\hline HR at 8 years & 0.37 & \\
\hline Race (referent = white) & 0.86 & 0.123 \\
\hline Sex $($ referent $=$ male $)$ & 0.89 & 0.365 \\
\hline \multicolumn{3}{|l|}{ Mode of transmission } \\
\hline MSM & 1.00 & Reference \\
\hline IDU & 0.97 & 0.803 \\
\hline No identified risk & 1.00 & 0.977 \\
\hline Other & 0.93 & 0.606 \\
\hline
\end{tabular}


utilization of health care model (Table 2), the ratio of ER visits to the total number of ER and outpatient visits was significantly associated with survival time $(p<0.001)$. Also, in this model, white race was significantly associated with increased probability of survival $(\mathrm{HR}=$ $0.79, p=0.020)$.

$t$-Tests were used to test for colinearity between the perceived access and utilization indicators. Colinearity was detected between the utilization indicator (ER visit ratio) and two of the three indicators of access to health care (not seeking care because of inability to pay and health insurance status). A lower ER visit ratio indicated that a smaller proportion of health care visits occurred in the ER than in the outpatient clinic. The ER visit ratio was lower for those who did not report financial barriers to seeking care than for those who did not seek care because of inability to pay. The mean ER visit ratio was 0.17 [standard deviation (SD) = 0.24] for those who did not avoid seeking health care due to an inability to pay versus 0.27 (SD $=0.31$ ) for those who did avoid seeking health care because they could not pay, and the $t$-test had a $p$ value $=0.019$. For those who had health insurance, the ER visit ratio was lower than for those who did not have insurance. The mean ER visit ratio was 0.17 (SD = 0.24 ) for those with health insurance versus
$0.29(\mathrm{SD}=0.33)$ for those who did not have health insurance, and the $t$-test $p$-value $=0.011$.

\section{DISCUSSION}

In this study we examined the association between survival after AIDS and indicators of perceived access to health care and utilization of health services. We observed that the indicator for utilization of health care and race (in the utilization model only) were significantly associated with survival after AIDS, but that sex, mode of transmission, and perceived access to health care were not significantly associated with survival in the study population. These results affirm the hypothesis posed by other researchers that utilization of health care services was an underlying source of differences in survival previously attributed to race, sex, or mode of transmission. ${ }^{1,2}$

HAART was a significant predictor of survival in both models. This is consistent with previous studies that found HAART to be associated with decreased morbidity and mortality in HIV-infected populations. ${ }^{7-11}$ We also found that the association between HAART and survival decreased over time after AIDS. We attribute this to the long-term non-progressors who were infected prior to the avail-

Table 2. Utilization of Health Care

\begin{tabular}{lc}
\hline & $\begin{array}{c}\text { Hazard ratio }(\text { HR }) \\
(\mathrm{n}=752 ; 552 \text { deaths })\end{array}$ \\
Predictor variable & 2.04 \\
\hline ER visit ratio (ER visits/total ER + outpatient visits) & $<0.001^{\mathrm{b}}$ \\
HAART (referent = HAART) & $0.001^{\mathrm{b}}$ \\
Time dependent (HAART $\times$ time) & 0.12 \\
HR at 1 year & 0.14 \\
HR at 2 years & 0.22 \\
HR at 5 years & 0.35 \\
HR at 8 years & 0.79 \\
Race (referent = white) & 0.86 \\
Sex (referent = male) & 1.00 \\
Mode of transmission & 0.83 \\
MSM & 0.99 \\
IDU & 0.89 \\
No identified risk & $0.025^{\mathrm{c}}$ \\
Other & 0.262 \\
\hline
\end{tabular}

${ }^{\mathrm{a} C o x}$ proportional hazards model for the relative hazard of death in AIDS patients.

$\mathrm{b}_{p}<0.0005$.

${ }^{\mathrm{c}} p<0.05$.

HAART, highly active antiretroviral therapy; MSM, men who have sex with men; IDU, injection drug users; ER, emergency room. 
ability of HAART and lived without HAART for many years, thus narrowing the gap in survival time between the two treatment groups. This finding is reminiscent of the mortality crossover effect discussed by Wing and colleagues. ${ }^{26}$ They described mortality ratios that change over time with the force of mortality operating earlier in some populations than in others. Over time, the healthy and resilient of a group remain alive, and the mortality ratio eventually reaches unity.

Individual perception of access to health care was not associated with survival. Perceived access to health care was measured using three indicator questions. The indicators that we used to measure perceived access to health care may have been inadequate. Andersen and colleagues discuss the dimensions of potential and realized access to medical care and several indicators of these dimensions. ${ }^{27}$ The perceived access indicators that we used fall under the category of potential access and are not complete according to their guide. This may explain the lack of association between perceived access to care and survival that we found. Since all of our study participants have already accessed the health care system, utilization of these services may better predict survival.

Utilization of health care services was measured as the ratio of ER visits to the total number of ER and outpatient visits. Previous studies found that HIV-infected patients had a much better survival probability if they received regular care from providers who specialized in treating HIV infection, compared to providers who did not specialize in HIV care. ${ }^{28,29}$ Our results were consistent with these findings. Individuals who received a greater proportion of their care in the ER had a worse survival prognosis than those who received more outpatient care (which was provided by physicians who specialized in HIV infection).

Perceived access and utilization (ER visit ratio) were not independent indicators of health care access. Those without insurance went to the ER more often, and were less likely to have a regular source of care. Similarly, the group that did not seek medical care due to inability to pay received a greater proportion of their care from the ER. Because of the colinearity that was detected, we expected that both perceived access and uti- lization would significantly predict survival. Since this was not the case, we conclude that the access to care indicators were inadequate.

There are several limitations of this study. First, the ASD database relies on what was recorded in medical records at the two study sites to completely represent the history of each patient's HIV care. Patients may have been seen for HIV care at additional facilities and that information would not be available. HAART misclassification may have occurred in cases where HAART was prescribed at non-ASD facilities, and not included in the medical records at the ASD facilities.

A second limitation is that SHAS interviews were done only once and at varying times throughout each persons disease process, which may have caused additional bias in the study. However, by limiting SHAS variables to those which should not change over time (e.g., race), or change minimally (e.g., access to health care and utilization of health services) such bias was minimized. Additionally, in the survival analysis models data were left truncated taking into consideration when the SHAS interview occurred. This method adjusts for the bias that would result from not including those who died before they had the opportunity to be interviewed.

A third limitation to the study is that the selection of SHAS participants was not random. However, all eligible participants were approached and the refusal rate was low (2\%). Therefore, this should not have a significant impact on study outcomes.

A fourth limitation is the possibility that those with a higher proportion of ER visits were sicker. This would cause them to have worse survival probability. We attempted to minimize the resulting bias by including only individuals who met the criteria for AIDS, thus presuming high levels of morbidity in the entire population.

Populations that are historically underserved by the health care system, such as blacks, women, and injection drug users, are also at high risk for HIV infection. Cunningham and colleagues found that access to medical care was poorer for those with HIV than for other chronic diseases. ${ }^{4}$ Consistent with our findings, other studies have shown that even among in- 
dividuals within the health care system, a regular source of health care (including clinic appointments with HIV specialists) increases survival time for people living with AIDS. ${ }^{28,29}$ It is crucial to provide HIV-infected populations with tools that will enable them to adequately access a regular source of health care in a way that will most benefit their health.

\section{ACKNOWLEDGMENTS}

This project was supported by a cooperative agreement from the Centers for Disease Control and Prevention and the Michigan Department of Community Health. The authors thank Drs. Jonathan Cohn and Rodger MacArthur from the Detroit Medical Center, Drs. Norman Markowitz and Douglas Mayers from the Henry Ford Health System, the ASD data abstraction and data entry teams, and the SHAS interviewers.

\section{REFERENCES}

1. Melnick SL, Sherer R, Louis TA, et al. Survival and disease progression according to gender of patients with HIV infection: The Terry Beirn Community Programs for Clinical Research on AIDS. IAMA 1994;272(24):1915-1921.

2. Curtis JR, Patrick DL. Race and survival time with AIDS: A synthesis of the literature. Am J Public Health 1993;83(10):1425-1428.

3. Cunningham WE, Hays RD, Williams KW, et al. Access to medical care and health-related quality of life for low-income persons with symptomatic human immunodeficiency virus. Med Care 1995;33(7):739-754.

4. Cunningham WE, Hays RD, Ettl MK, et al. The prospective effect of access to medical care on healthrelated quality-of-life outcomes in patients with symptomatic HIV disease. Med Care 1998;36(3):295-306.

5. Centers for Disease Control and Prevention. HIV/ AIDS Surveillance Report 1999;11(1).

6. Michigan Department of Community Health. Michigan HIV/AIDS Year End Review January, 2000.

7. Hogg RS, Yip B, Kully C, et al. Improved survival among HIV-infected patients after initiation of tripledrug antiretroviral regimens. Can Med Assoc J 1999;160(5):659-665.

8. Detels R, Munoz A, McFarlane G, et al. Effectiveness of potent antiretroviral therapy on time to AIDS and death in men with known HIV infection duration. JAMA 1998;280(17):1497-1503.

9. Michaels SH, Clark R, Kissinger P. Declining mor- bidity and mortality among patients with advanced human immunodeficiency virus infection. $\mathrm{N}$ Engl J Med 1998;339(6):405-406.

10. Palella FJ Jr, Delaney KM, Moorman AC, et al. Declining morbidity and mortality among patients with advanced human immunodeficiency virus infection. N Engl J Med 1998;338(13):853-860.

11. Brodt HR, Kamps BS, Helm EB, et al. Kaposi's sarcoma in HIV infection: impact on opportunistic infections and survival. AIDS 1998;12(12):1475-1481.

12. Farizo KM, Buehler HW, Chamberland ME, et al. Spectrum of disease in persons with human immunodeficiency virus infection in the United States. JAMA 1992;267(13):1798-1805.

13. Buehler JW, Diaz T, Hersch BS, et al. The supplement to HIV-AIDS Surveillance Project: An approach for monitoring HIV risk behaviors. Public Health Rept 1996;111(Suppl 1);133-137.

14. Centers for Disease Control and Prevention. Guidelines for the use of antiretroviral agents in HIV-infected adults and adolescents. Morb Mortal Wkly Rep 1998;47:RR-5.

15. Centers for Disease Control and Prevention. 1993 revised classification system for HIV infection and expanded case definition for AIDS among adolescents and adults. Morb Mortal Wkly Rep 1992;41RR-17.

16. Centers for Disease Control and Prevention. HIV/ AIDS Reporting System (HARS) User Manual: Version 2.0 Atlanta, GA: CDC, 1995.

17. Pehrson PO, Lindback S, Lidman $C$, et al. Longer survival after HIV infection for injecting drug users than for homosexual men: Implications for immunology. AIDS 1997;11:1007-1012.

18. Prins M, Veugelers PJ. Comparison of progression and non-progression in injecting drug users and homosexual men with documented dates of HIV-1 seroconversion. AIDS 1997;11:621-631.

19. Scott WK, Sy FS, Jackson KL, et al. Survival after AIDS diagnosis in South Carolina. J South Carolina Med Assoc 1997;93(1):5-12.

20. Blum S, Singh TP, Gibbons J, et al. Trends in survival among persons with acquired immunodeficiency syndrome in New York City. The experience of the first decade of the epidemic. Am J Epidemiol 1994;139: 351-361.

21. Rothenberg R, Woelfel M, Stoneburner R, et al. Survival with the acquired immunodeficiency syndrome: Experience with 5833 cases in New York City. N Engl J Med 1987;317:1297-1302.

22. Allison PD. Survival analysis using the SAS System: A practical guide. Cary NC: SAS Institute, 1995.

23. Grambsch PM, Therneau TM. Proportional hazards tests and diagnostics based on weighted residuals. Biometrika 1994;81:515-526.

24. SAS Institute Inc. SAS/STAT Software: Changes and enhancements for release 6.12. Cary, NC: SAS Institute;1996.

25. Statistical Sciences. S-PLUS guide to statistical and mathematical analysis, version 3.2. Seattle: Statistical Sciences, 1993. 
26. Wing S, Manton KG, Stallard E, Hames CG, Tryoler HA. The black/white mortality crossover: investigation in a community-based study. J Gerontol 1985; 40(1):78-84.

27. Andersen RM, McCutcheon A, Aday LA, et al. Exploring dimensions of access to medical care. Health Serv Res 1983;18(1):49-74.

28. Battegay M, Wirz M, Steuerwald MH, et al. Early participation in an HIV cohort study slows disease progression and improves survival. J Intern Med 1998; 244:479-487.

29. Laine C, Markson LE, McKee LJ, et al. The relation- ship of clinic experience with advanced HIV and survival of women with AIDS. AIDS 1998;12(4):417-424.

Address reprint requests to: JoLynn Pratt Montgomery Herman Kiefer Health Complex \#210B 1151 Taylor Detroit, MI 48202

E-mail: montgomeryjp@michigan.gov 
This article has been cited by:

1. Neil S. Wenger, Roy T. Young. 2007. Quality Indicators for Continuity and Coordination of Care in Vulnerable Elders. Journal of the American Geriatrics Society 55:s2, S285-S292. [CrossRef]

2. Catherine Kaukinen, Christopher Fulcher. 2006. Mapping the social demography and location of HIV services across Toronto neighbourhoods. Health and Social Care in the Community 14:1, 37-48. [CrossRef]

3. Noya Galai, David Vlahov, Joseph C. Bareta, Cunlin Wang, Sylvia Cohn, Timothy R. Sterling. 2005. Prognostic Factors for Survival Differ According to CD4+ Cell Count Among HIV-Infected Injection Drug Users. JAIDS Journal of Acquired Immune Deficiency Syndromes 38:1, 74-81. [CrossRef]

4. Ka-Hing Wong, Shui-Shan Lee, Kelvin Hon-Kei Low, Wai-Yee Wan . 2003. Temporal Trend and Factors Associated with Late HIV Diagnosis in Hong Kong, a Low HIV Prevalence Locality Temporal Trend and Factors Associated with Late HIV Diagnosis in Hong Kong, a Low HIV Prevalence Locality. AIDS Patient Care and STDs 17:9, 461-469. [Abstract] [PDF] [PDF Plus]

5. Sara A. Mirza, Maureen Phelan, David Rimland, Edward Graviss, Richard Hamill, Mary E. Brandt, Tracie Gardner, Matthew Sattah, Gabriel Ponce de Leon, Wendy Baughman, Rana A. Hajjeh. 2003. The Changing Epidemiology of Cryptococcosis: An Update from Population-Based Active Surveillance in 2 Large Metropolitan Areas, 1992-2000. Clinical Infectious Diseases 36:6, 789-794. [CrossRef] 